\title{
Adapted Magnetic Wheel Unit for Compact Robots Inspecting Complex Shaped Pipe Structures
}

\author{
Fabien Tâche*, Wolfgang Fischer*, Roland Moser ${ }^{\dagger}$, Francesco Mondada ${ }^{\ddagger}$, Roland Siegwart* \\ *Autonomous Systems Lab (http://www.asl.ethz.ch), Eidgenössische Technische Hochschule Zürich (ETHZ), \\ 8092 Zürich, Switzerland \\ ${ }^{\dagger}$ ALSTOM (Switzerland) Ltd, ALSTOM Power Service (http://www.alstom.com), \\ 5401 Baden, Switzerland \\ ${ }^{\ddagger}$ Laboratoire de Systèmes Robotiques (http://lsro.epfl.ch), Ecole Polytechnique Fédérale de Lausanne (EPFL), \\ 1015 Lausanne, Switzerland \\ Email:ftache@ethz.ch,wfischer@ethz.ch,rsiegwart@ethz.ch,roland.moser@power.alstom.com, \\ francesco.mondada@epfl.ch
}

\begin{abstract}
This paper describes a novel magnetic wheel unit integrating a mechanism that can be used for lifting and stabilizing the unit. The mechanism consists of 2 active lever arms mounted on each side of the wheel and rotating coaxially with the wheel. This mechanism allows slightly lifting the magnetic wheel at any desired position on the wheel circumference and consequently decreasing the magnetic force at this specific location. The same mechanism can also be used to stabilize the wheel, when external forces are unfavorable. This paper also describes the potential of this concept for in-pipe inspection technologies. Indeed it can be used to increase the mobility of magnetic wheels robots which are currently not able to negotiate complex obstacles. At the same time, it allows building smaller robots, since the self stabilizer system allows reducing the amount of required magnetic wheels to only two units.
\end{abstract}

Index Terms-Magnetic wheel, in-pipe inspection, climbing, obstacle passing.

\section{INTRODUCTION}

Typical pipe constructions such as sewers, gaz/oil transmission pipelines, gaz/oil distribution pipes are suffering from several diseases when getting old. Aging, corrosion and mechanical stress generally lead to the loss of material thickness or generation of cracks that can cause leakages or sometimes the destruction of the pipe construction.

Thus, periodic inspection of the pipe system is required in order to prevent such damages. Since many of these constructions have not been designed to optimize automatic inspection and repair tasks, inspection and maintenance generate huge costs, especially if disassembling or even excavating is necessary. Inspection and maintenance technology has then become a growing industry market and a large variety of systems have been developed. Several recent reports [1], [2] have already established a state of the art of the existing industrial and academic systems, by classifying and comparing their performances.

As summary, Roh [3] proposes a classification of the possible locomotion strategies used to solve the in-pipe inspection problems: robots may be pig type (passively driven by the fluid pressure), wheel type, caterpillar type, wall-pressed type, walking type, inchworm type or screw type. Bends are usually negotiated thanks to differential-drive steering (for single body systems) or articulated structures.

When climbing ability is required, the most common solution is to use spreading systems [3]-[9] as for robots depicted in Figure 1.

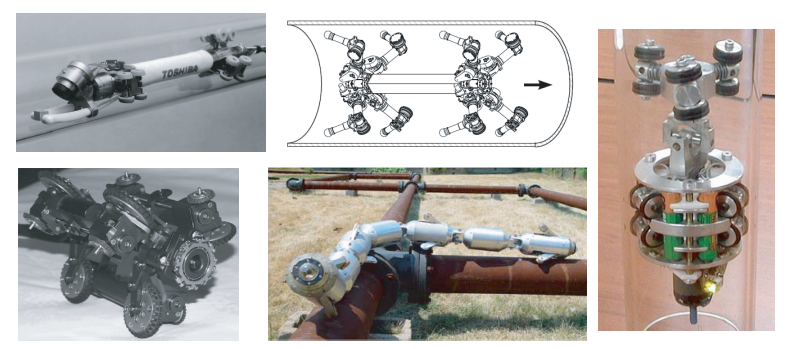

Fig. 1. Locomotion systems for in-pipe inspection robots: Toshiba's robot [8], MORITZ [4], MRINSPECT IV [3], Explorer [9] and HELI-PIPE [6]

However, none of these systems can deal with narrow pipe environments which integrate high abrupt diameter changes, bends and also requires climbing ability. In this case, it is interesting to combine the locomotion system with attachment elements such as grasps, suction cups [10], adhesive polymers [11] or (electro)magnetic elements. These technics are typically used in wall climbing robots for tank inspection [12] or wall cleaning tasks [10]. Since the first concepts usually imply complex mechanics and that the considered environment is ferromagnetic, magnetic attachment systems were selected for this application. This overview of existing systems then focuses on magnetic wheel solutions.

The idea of using magnetic elements or wheels on mobile robots is not new, since Guy [13] patented concepts for magnetic wheels systems in 1972 already. Robots using magnetic elements for locomotion (wheels for Magnebots [14], Tripod [15] and Osaka Gas inspection robot [16] or tracks for Nanomag [17]) already exist. These robots take advantage of the magnetic force in order to travel on surfaces with any inclination regarding the gravity vector. Their mobility is however limited to smooth obstacle-free surfaces. By smooth, we refer to surfaces with high radii of curvature. 
In comparison, Kawaguchi [18], Yukawa [19] and Fischer [12] robots implemented special mechanisms to negotiate specific obstacles. The robot described in [12] and [19] are designed for vertical walls or the outer surfaces of pipes. These mechanisms aim for passing very difficult obstacles, but require a lot of space and many DOF. The robot from Kawaguchi [18] is more related to our application, since it is designed for inspecting the interior surfaces of pipes and uses a passive mechanism to overpass $90^{\circ}$ transitions.

Due to very restrictive environment constraints, the existing locomotion systems cannot be used in our specific application. This paper then describes a novel magnetic wheel unit that allows for designing compact and simple robots for inspecting narrow pipes with very complex shaped structures.

The paper is organized as follows. Section II introduces the challenging environment for which a mobile inspection robot has to be built and the specific requirements of the application. Section III analyzes existing solutions and shows their limitations. The necessity to use a magnetic element integrating a lifter mechanism is then explained in section IV. While several concepts and their limitations are described in section $\mathrm{V}$, an innovative adapted magnetic wheel unit is proposed in section VI. Finally this paper shows how this adapted magnetic wheel unit can help increasing the mobility of compact magnetic wheel robots.

\section{APPLICATION REQUIREMENTS}

As already mentioned in the introduction, among the existing in-pipe inspection systems, there does not exist any compact robot that can adapt to extreme diameter changes. As starting point for the discussion, this section first gives an overview of a specific application and the critical constraints of the environment.

\section{A. Environment Constraints}

Figure 2 shows the 3D CAD model of a typical environment that requires non destructive inspection (NDI). Here is a list of the most critical dimensions and characteristics of this environment:

- the wide range of inner diameters: the diameter varies from $200 \mathrm{~mm}$ (this defines the maximum robot space envelope) to $700 \mathrm{~mm}$ space envelope.

- the local abrupt inner diameter changes: up to $50 \mathrm{~mm}$ on Figure 2.

- it is composed of horizontal pipe elements, as well as vertical elements. Generally any inclination can be encountered.

- it is built out of ferromagnetic material.

\section{B. Robot purpose and required mobility}

Since the robot is intended for NDI, it has to embed typical NDI sensors such as cameras, Eddy current probes or electromagnetic acoustic transducer probes. Thus the robot should be able to carry its own weight plus some extra payload corresponding to the weight of NDI sensor and some manipulation tools.

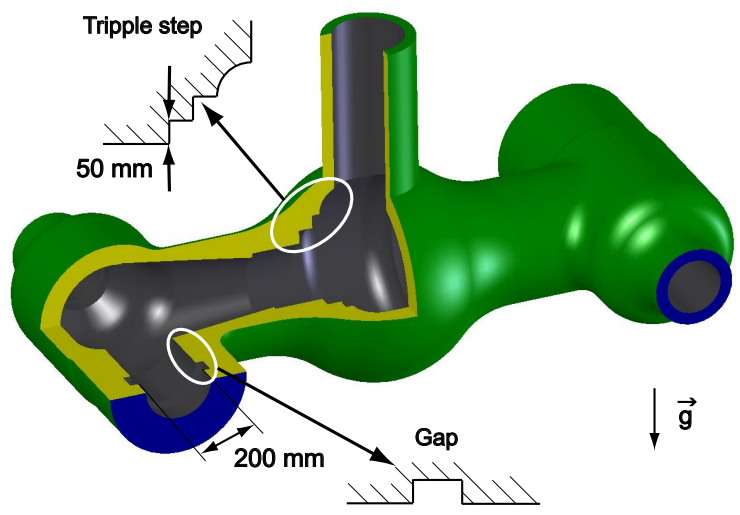

Fig. 2. 3D CAD model of a typical environment

Even if damages tend to appear in preferred locations, an efficient inspection robot needs a locomotion system that allows for bringing the inspection sensors to any location that might be damaged. The 3D CAD model represented on Figure 2 helps understanding the requirements of the locomotion system: the robot has to negotiate different transitions of $90^{\circ}$ convex and concave surfaces. For instance, the profile contains a triple step and a gap type obstacle. In addition to the obstacle complexity, the locomotion mechanism has to be independent of the gravity vector orientation. Indeed the same profile can be extracted from several points of view regarding gravity.

\section{ANALYSIS: MOBILITY, SIZE AND COMPLEXITY}

At first, it has to be verified that the assumption of using magnetic wheels as locomotion and attachment system is the best for this application. For this reason, existing systems are deeper analyzed and their limitations are presented.

\section{A. Necessity and optimality of magnetic wheels}

Since the robot has to work in a narrow in-pipe environment with vertical elements and $90^{\circ}$ bends, this drastically restricts the range of possible locomotion systems. As mentioned in the introduction, spreading systems is a common solution when climbing ability is required. An example is MORITZ [4] (Fig. 3), a crawler robot equipped with eight legs each equipped with two driven joints. MORITZ robot is able to crawl in tubes of any inclination and to manage curved pipes with diameter of $60-70 \mathrm{~cm}$. This robot weighs $20 \mathrm{~kg}$. Another example is Explorer [9] (Fig. 1), a seven-element articulated body robot, four of them being equipped with a set of three active deploying legs. This robot is able to drive in 6-in. and 8 -in. pipe elements and negotiate $45^{\circ}$ and $90^{\circ}$ bends. It can also drive on slightly inclined and short vertical pipe elements. Taking into account the mechanical and control complexity of legged robots and the complexity of implementing highly extensible legs in a restricted space envelope, these solutions have been discarded.

Analyzing the remaining attachment solutions, grasping is not an option in this environment, adhesive elements still have problems to keep their efficiency on long runs and suction 
cups do not seem optimal to work on curved surfaces. It seems rather obvious that complexity and size can be saved by taking advantage of the ferromagnetic environment and consequently using (electro)magnetic elements for attachment. Indeed, instead of having a robot that uses the whole available space in the pipe, it can be a compact unit that follows the profile of the pipe as illustrated in Fig. 3.

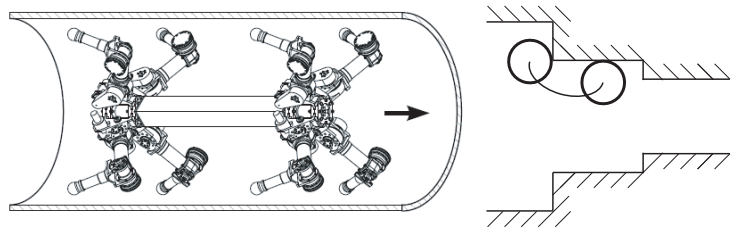

Fig. 3. MORITZ legged robot compared to the scheme of a compact magnetic wheel robot

\section{B. Magnetic wheels on passive articulation structures}

Given the problem of negotiating difficult obstacles, a good source of inspiration is the research on rough terrain exploration rovers. An excellent solution is to use a robot structure that passively adapts to the terrain shape and is able to negotiate obstacles larger than the wheel diameter. One could then first imagine equipping a Shrimp-like articulated structure [20] (Fig. 4) with magnetic wheels.

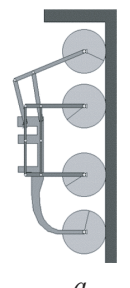

$a$

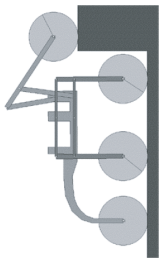

$b$

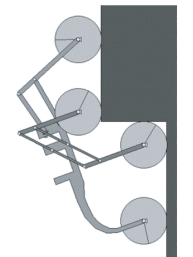

$c$

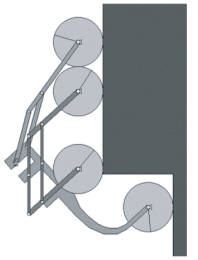

$d$
Fig. 4. Shrimp-like robot equipped with magnetic wheels

This type of solution does however not seem promising for several reasons:

- the complexity of downscaling and increasing the power to mass ratio of structures which integrate a high number of actuated wheels.

- on structures with a high number of wheels, wheel slippage is necessary for turning. Due to the high friction forces generated by magnetic wheels, these articulated structures are obviously not optimal.

- whereas the mechanisms in rough terrain robots only have to deal with the gravity, magnetic wheel climbing robots also have to get rid of the magnetic forces that are several times higher than the gravity.

\section{Magnetic wheels on active structures}

Active structures equipped with magnetic elements are proposed in [19] and [12]. They use active linear mechanisms, in order to lift off one or several wheels that pass over the obstacle (flange, valve or ridge) without rolling on it. These structures which have been developed for external pipe and gas tank inspection are not suitable for this application, since they are very complex (26, respectively 10 active degrees of freedom) and not compact enough for in-pipe inspection.

\section{ANALYSis of OBSTACLE NEGotiation}

As pointed out in the previous section, magnetic wheel climbing robots do not only have to deal with the gravity force, but also with the magnetic forces, especially when a wheel is in contact with 2 different surfaces. As illustrated in Figure 5 (left), the robot has to get rid of the magnetic force $F_{m a g \_1 b}$ when it drives away from the the vertical wall; whereas it has to get rid of the magnetic force $F_{\text {mag_1a }}$ when it drives away from the horizontal wall.
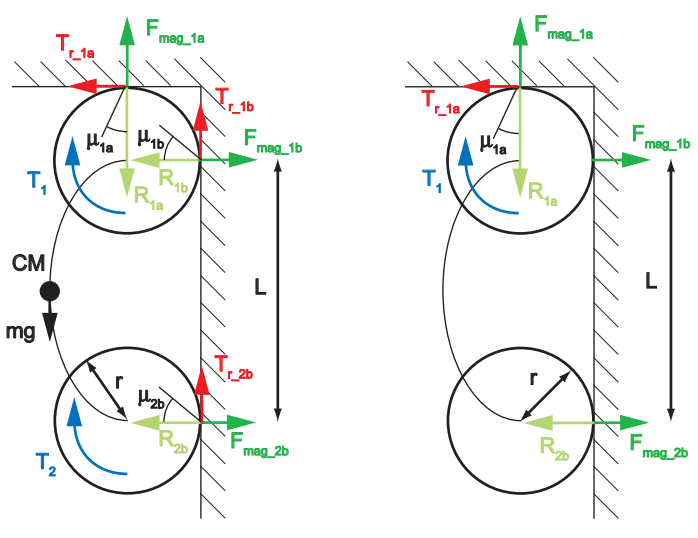

Fig. 5. Left: external forces and torques acting on a 2 pairs of motorized wheels system. Right: same model for a system with only front wheel traction. At the moment the wheel gets lifted, the traction $T_{r_{-} 1 b}$ and reaction $R_{1 b}$ forces tend to 0

A static model of the forces and torques (Fig. 5 left) acting on a 2 magnetic wheel-pairs system allows computing the friction coefficient and torques that are necessary to negotiate this $90^{\circ}$ concave obstacle. The necessity of using an adapted wheel can already be demonstrated by doing some simplifications (Fig. 5 right).

First, it is assumed that the robot with two pairs of wheels has only front wheel traction $\left(T_{2}=0\right)$. Secondly the weight $(\mathrm{mg})$ can be neglected, since magnetic wheel robots require magnetic forces several times higher than the robot's weight in order to ensure a good security against falling. Getting rid of the unwanted magnetic force $\left(F_{\text {mag_1b }}\right)$ requires a traction force $\left(T_{r_{-} 1 a}\right)$ bigger than $F_{m a g_{-} 1 a} \times L /(L+r)$, respectively a friction coefficient $\mu_{1 a}$ bigger than $L /(L+r)$. Indeed we assume that the traction $T_{r_{-} 1 b}$ and reaction $R_{1 b}$ forces tend to zero at the moment the wheel lifts off. Consequently, for optimization, $L / r$ ratio has to be decreased as much as possible. Figure $6 a$ shows the case of $L=r$ for which the required $\mu_{1 a}$ would be 0.5 . Detailed calculations (computer simulation) without this simplification show similar results.

The system length should however not be too short, in order to avoid the singular case $b$, for which the system gets blocked on a convex obstacle. Indeed traction force cannot be provided, 


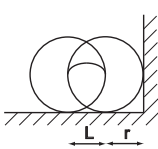

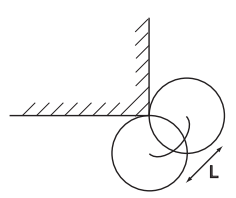

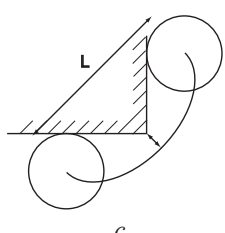

Fig. 6. $a$ : 2 pairs of wheels configuration with $L=r, b$ : singularity on a convex obstacle, $c$ : ground clearance problem for a long system

since the effective distance between wheel contact points is zero. $L$ has then to be bigger than $\sqrt{2} * r$ to avoid this singularity on convex edges. Increasing too much this dimension has also a drawback regarding ground clearance (Fig. 5c).

From these 2 simple examples, we already notice the difficulty to implement a unique system adapted to different scenarios. This problem has even no solution, if the arrangement and size of these obstacles gets more complex as illustrated on Figure 7 , especially in case $c$ for which all wheels have two contacts with the terrain. This clearly shows the necessity to use a system which decreases or cancels the unwanted magnetic force, in order to go towards the implementation of a universal system able to face any combination of $90^{\circ}$ surface transitions.

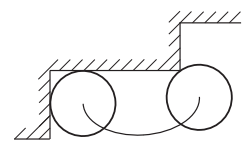

$a$

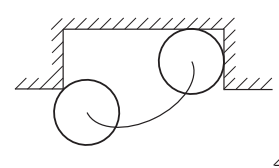

$b$

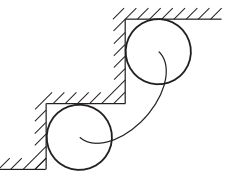

Fig. 7. Complex shaped obstacles: double steps $(a)$, gap shorter than the robot length $(b)$, double steps, all wheels with double contact points $(c)$

\section{CONCEPTS FOR ADAPTED WHEEL UNIT}

After having discussed the necessity of having an adapted wheel, this section proposes concepts for such magnetic wheel units: first passive concepts, as well as active ones.

\section{A. Passive magnetic wheel}

By passive magnetic wheel, we refer to wheels that passively adapt their magnetic property regarding the shape of the terrain. An example is the wheel proposed in [18]. Picture 8 reminds the mechanical principle of the inner magnetic wheel which rolls on the outer ferromagnetic tire. The magnetic force is then smoothly transferred from one surface to the other. In this paper, the reliability of the system (risk of loosing contact on the ceiling) is however not discussed. Indeed position $c$, for which the magnetic force is spread between the two contact points (one being an edge), may be critical.

\section{B. Active magnetic wheel}

On the contrary, by active magnetic wheel, we refer to wheels which actively modify their magnetic property in order to adapt to the terrain.

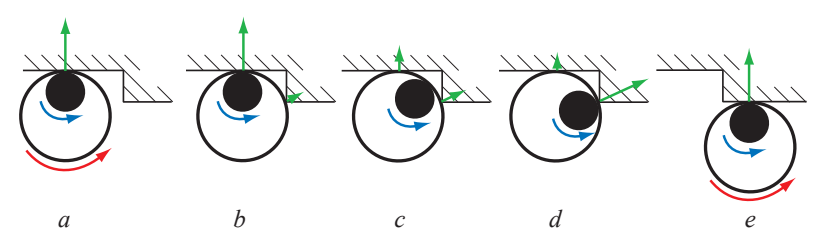

Fig. 8. Passive wheel mechanics as proposed in [18]. Arrows show the movement of the inner wheel (blue) and outer tire (red) as well as the evolution of the magnetic force (green)

1) Global force modulation: Replacing the central ring magnet by an active coil allows modulating the magnetic force of the wheel. A first drawback of this electromagnetic solution is that it does modulate the force in the same way on the whole wheel circumference: this is a kind of global force modulation system. Not only the unwanted magnetic force is reduced, but also the magnetic force that is necessary for traction and adhesion: the robot may fall when passing obstacles on the ceiling. Another major drawback is that a failure on the power supply would cancel the magnetic force and would cause the robot's fall.

2) Local force modulation: Due to the drawbacks of global force modulation systems mentioned above, local force modulation systems are analyzed in more details. The most promising concepts for such systems are illustrated in Figure 9. In concept $a$, the permanent magnet is replaced by active coils distributed on the wheel circumference. Decreasing the current in the correct coil allows for diminishing the unwanted magnetic force. Concept $b$ consists in using a linear mechanism that moves axially in order to lift the wheel. Yukawa [19] implemented this solution on the Type III robot. Concept $c$ consists in using a rotary mechanism, rotating coaxially with the wheel, which gets tangentially into contact with the terrain.

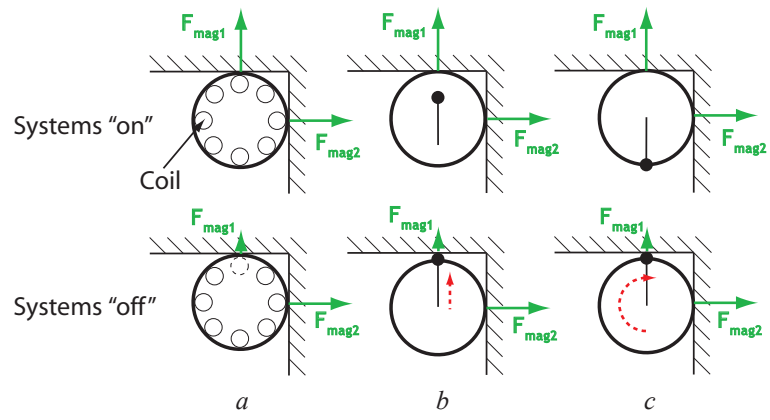

Fig. 9. Active magnetic wheel: electromagnetic $(a)$, linear lifter $(b)$ and rotary lifter $(c)$

The linear lifter $b$ only works when the unwanted magnetic contact point is always located at the same position. Since in our complex shaped environment, the contact point with the terrain can be anywhere on the wheel circumference, this concept does not make sense. Due to the complexity (wiring) and low efficiency of electromagnetic wheels (lower energy density than powerful magnets), this concept is discarded. Concept $c$ seems to be the most promising and is further investigated. 


\section{AdAPTED MAGNETIC WHEEL UNIT}

This chapter describes the innovative magnetic wheel unit integrating an active rotary lifter mechanism. Figure 10 shows a CAD model of a possible implementation of the mechanism. The lifter mechanism consists in two powered rotating lever arms mounted on each side of the wheel. The rotation axis of these arms is common to the wheel rotation axis, but is independently actuated. These arms have two complementary functions: they can either be used to lift off the wheel in order to decrease the force at the unwanted contact point ("lifter") or to stabilize it laterally ("stabilizer").

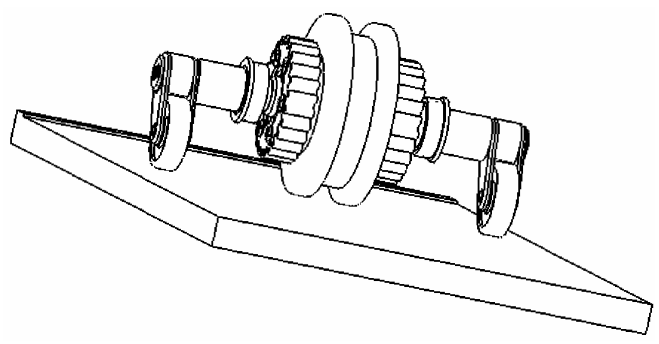

Fig. 10. CAD model of a rotary lifter mechanism. Wheel and lifter are independently actuated through two different gears mounted on a common axis

\section{A. Wheel arms as lifter}

As mentioned, these arms can be used to lift off the wheel, in order to decrease the magnetic force at the unwanted contact point on the circumference as illustrated on Figure 11. The imbalance between the magnetic forces $F_{m a g 1}$ and $F_{\text {mag } 2}$ then solves the problem pointed out in section IV. This imbalance can be modulated, depending if the lifter is applied perpendicularly (Fig.11 left) to the ground or not (Fig.11 right).
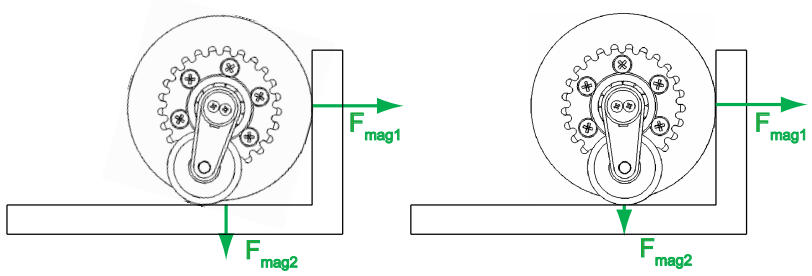

Fig. 11. The lever arm mechanism is applied, in order to slightly lift off the wheel and decrease the magnetic force at the unwanted contact point

The unwanted magnetic force $\left(\mathrm{F}_{\text {mag } 2}\right.$ in this example) can even be fully canceled if the wheel is sufficiently lifted off. It is however safer to design it, so that an offset force remains when the lifter is applied perpendicularly to the surface. Indeed it prevents the wheel to fully lose magnetic attraction force, in case the lifter is applied, when the wheel is in contact with only one ferromagnetic surface.

Since the lifter can reach any position on the wheel circumference, this adapted magnetic wheel allows for a 2 wheelpairs robot negotiating step obstacles. In the sequence of movement (Figure 12), it can be remarked that the lifters of the front and rear wheels are not applied at the same position, regarding the robot (a reference position is marked by a dashed line).

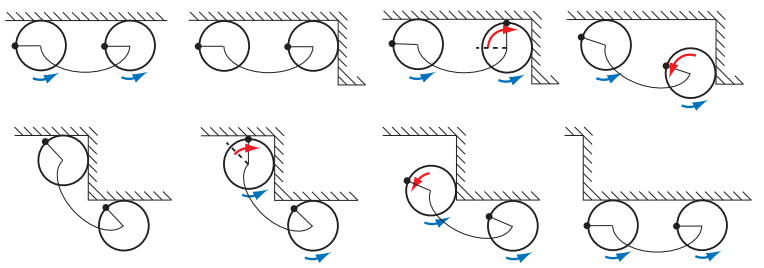

Fig. 12. Robot negotiating a step obstacle

This mechanism does not only allow for negotiating simple step obstacles, it even helps negotiating the most difficult type of obstacles (Fig.2), respectively when all wheels are in double contact with the terrain. This is illustrated in Figure 13, on which a 2 wheel-pairs robot uses the lifter mechanism on both wheel pairs at the same time.

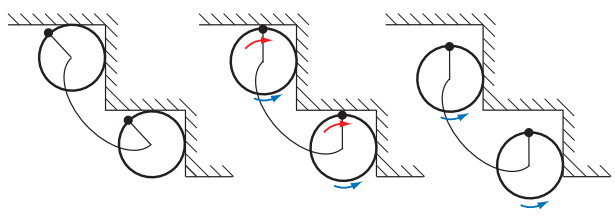

Fig. 13. Robot negotiating a double step obstacle

\section{B. Wheel arms as stabilizer}

As mentioned, the $2^{\text {nd }}$ functionality of this magnetic wheel lifter implementation is that the two lever arms can also be used as wheel stabilizer when the gravity vector is unfavorable, as shown on Figure 10. This feature might be seen as useless, if one thinks to 4 wheels robots with Ackermann steering geometry or differential-drive type robots that are laterally self stabilized. However we claim that this new system has a great advantage considering magnetic wheel robots miniaturization, thanks to the possibility to reduce the locomotion concept to 2 aligned adapted wheel units.
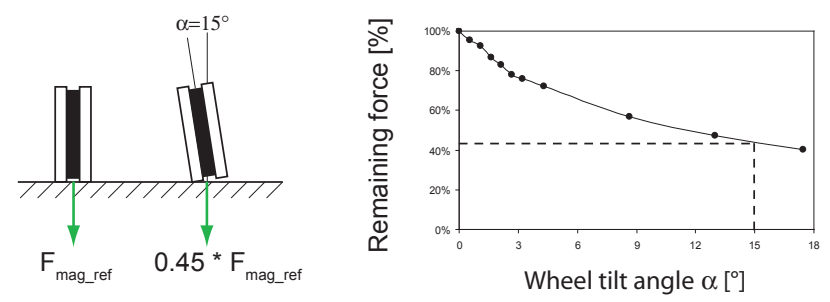

Fig. 14. Magnetic force of the magnetic wheel regarding wheel tilt angle

When dealing with the curved surfaces of internal pipes, an important constraint is to take care that the wheel provide their maximal magnetic force. For a standard wheel, this condition 
is reached when it stands perpendicularly to the surface. Indeed some tests showed that the magnetic force decreases more than $50 \%$ when the wheel is only tilted $15^{\circ}$ from its optimal orientation (Fig.14).

A partial solution to this problem is to build wheels with an adapted geometry that fit a certain range of pipe diameters as proposed by Kawaguchi [18]. Nevertheless the optimal solution consists in implementing a mechanical system that allows passively adapting the wheel orientation to the terrain configuration.

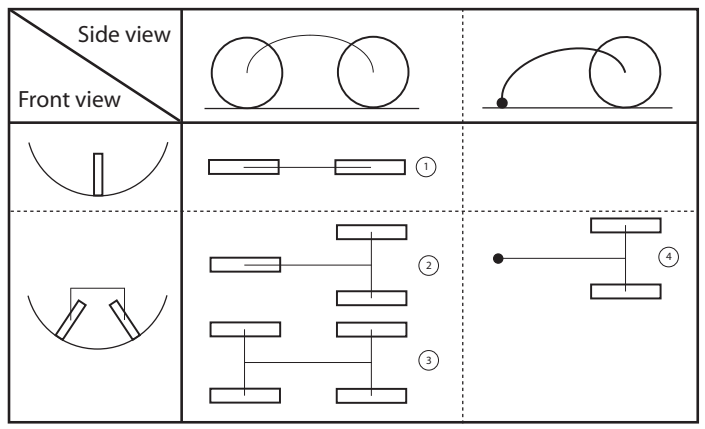

Fig. 15. 2 to 4 wheels configurations: matrix of top view regarding side and front views

By analyzing standard wheel configurations for robots with at most 4 wheels (Figure 15), we notice that arrangements (2), (3) and (4) require this type of mechanism. The advantage of this lifter arm implementation is then obvious: using them as lateral stabilizer allows designing a system with 2 aligned wheels (1) which is compact and simple.

\section{CONCLUSION AND FUTURE WORK}

After having presented an overview of the existing locomotion concepts for in-pipe inspection, this paper first describes an application that requires a compact system that can deal with high abrupt diameter changes and vertical climbing. It shows that magnetic wheels are the best concept for this ferromagnetic environment. It is then shown why an adapted magnetic wheel is necessary in order to negotiate the complex obstacles of the considered application.

A promising solution to this locomotion problem is then presented: an innovative active magnetic wheel unit incorporating an active lifter-stabilizer mechanism. It is shown how this locomotion concept can be used in order to negotiate complex shaped structures. The paper finally presents its advantages towards robot downsizing and simplification, by explaining that a 2 aligned wheels structure is sufficient.

Ongoing work consists in implementing a prototype robot integrating this mechanism, in order to validate the concept. Then a control strategy will have to be proposed in order to control the active system.

\section{ACKNOWLEDGMENTS}

We would like to thank ALSTOM for supporting this work as exploration research.

\section{REFERENCES}

[1] B.Bruce, R.Gordon, M.Sullivan, and C.Neary, "Internal repair of pipelines - technology status assessment report," Edison Welding Institute, Columbus, USA, Tech. Rep. DE-FC26-02NT4163, Nov. 2002.

[2] H.Schempf, "In-pipe-assessment robot platforms - phase I - state-ofthe-art review," Canergie Mellon University, Pittsburgh, USA, Report to National Energy Technology Laboratory REP-GOV-DOE-20041102, Nov. 2004.

[3] S. Roh and H. R. Choi, "Differential-drive in-pipe robot for moving inside urban gas pipelines," IEEE Transactions on Robotics, vol. 21, no. 1, pp. 1-17, Feb. 2005

[4] A. Zagler and F. Pfeiffer, "MORITZ a pipe crawler for tube junctions," in Proc. of the 2003 IEEE International Conference on Robotics \& Automation (ICRA'03), Taipei, Taiwan, Sept. 2003, pp. 2954-2959.

[5] M.Muramatsu, N.Namiki, R.Koyama, and Y.Suga, "Autonomous mobile robot in pipe for piping operations," in Proc. of the 2000 IEEE/RSJ International Conference on Intelligent Robots and Systems (IROS'2000), Takamatsu, Japan, Oct. 2000, pp. 2166-2171.

[6] M. Horodinca, I. Doroftei, E. Mignon, and A. Preumont, "A simple architecture for in-pipe inspection robots," in International Colloquium on Mobile and Autonomous Systems, Magdeburg, Germany, June 2002.

[7] S. Hirose, H. Ohno, T. Mitsui, and K. Suyama, "Design of in-pipe inspection vehicles for $\varnothing 25, \varnothing 50, \varnothing 150$ pipes," in Proc. of the 1999 IEEE International Conference on Robotics and Automation (ICRA'99), Detroit, USA, May 1999, pp. 2309-2314.

[8] K. Suzumori, T. Miyagawa, M. Kimura, and Y. Hasegawa, "Micro inspection robot for 1-in pipes," IEEE/ASME Transactions on Mechatronics, vol. 4, no. 3, pp. 286-292, Sept. 1999.

[9] H. Schempf, E. Mutschler, V. Goltsberg, G. Skoptsov, A. Gavaert, and G. Vradis, "Explorer: Untethered real-time gas main assessment robot system," in Proc. of the $1^{\text {st }}$ International Workshop on Advances in Service Robotics (ASER'03), Bardolino, Italy, Mar. 2003.

[10] F.Cepolina, R.C.Michelini, R.P.Razzoli, and M.Zoppi, "Gecko, a climbing robot for walls cleaning," in International Workshop on Advances in Service Robotics (ASERO3), Bardolino, Italy, Mar. 2003.

[11] M. Greuter, G. Shah, G. Caprari, F. Tâche, R. Siegwart, and M. Sitti, "Toward micro wall-climbing robots using biomimetic fibrillar adhesives," in Proc. of the International Symposium on Autonomous Minirobots for Research and Edutainment (AMIRE'2005), Fukui, Japan, Sept. 2005.

[12] W.Fischer, F.Tâche, and R.Siegwart, "Magnetic wall climbing robot for thin surfaces with specific obstacles," in Proc. of the International Conference on Field and Service Robotics (FSR'07), Chamonix, France, July 2007.

[13] W.Guy, "Magnetic wheel," U.S. Patent 3690393 , Sept. 12, 1972

[14] A. H.Slocum, S. Awtar, and J. Hart, "Magnebots - a magnetic wheels based overhead transportation concept," in Proc. of the $2^{\text {nd }}$ IFAC Conference on Mechatronics Systems, Berkeley, USA, Dec. 2002.

[15] Jireh Industries LTD. (2007, July) Jireh industries LTD. [Online]. Available: http://www.jireh-industries.com

[16] T. Sogi, Y. Kawaguchi, H. Morisaki, K. Ohkawa, N. Kai, and H. Hayakawa, "Inspection robot for spherical storage tanks," in Proc. of the $26^{\text {th }}$ International Conference on Industrial Electronics Control and Instrumentation (IECON'2000), Nagoya, Japan, Oct. 2000, pp. 393-398.

[17] Inuktun. (2006, July) Inuktun. [Online]. Available: http://www.inuktun.com

[18] Y.Kawaguchi, I.Yoshida, H.Kurumatani, T.Kikuta, and Y.Yamada, "Internal pipe inspection robot," in Proc. of the IEEE International Conference on Robotics and Automation (ICRA'95), Nagoya, Japan, May 1995, pp. 857-862.

[19] T. Yukawa, H. Okano, and S. Komatsubara, "Mechanisms for the movement of piping inspection robot with magnetic elements," in Proc. of the Sixth IASTED International Conference on Robotics and Applications (RA'05), Cambridge, USA, Nov. 2005.

[20] T.Estier, Y.Crausaz, B.Merminod, M. Lauria, R. Piguet, and R. Siegwart, "An innovative space rover with extended climbing abilities," in Proc. of Space and Robotics, Albuquerque, USA, Feb. 2000. 